\title{
La littératie familiale et les habiletés en conscience phonologique des enfants de maternelle
}

\section{Family Literacy and Phonological Awareness in Kindergarten Students}

\section{La literacia familiar y las habilidades en conciencia fonológica de los niños de preescolar}

\section{Monica Boudreau, Lise Saint-Laurent et Jocelyne Giasson}

Volume 34, numéro 2, automne 2006

L’éveil à l'écrit

URI : https://id.erudit.org/iderudit/1079028ar

DOI : https://doi.org/10.7202/1079028ar

Aller au sommaire du numéro

Éditeur(s)

Association canadienne d'éducation de langue française

ISSN

0849-1089 (imprimé)

1916-8659 (numérique)

Découvrir la revue

Citer cet article

Boudreau, M., Saint-Laurent, L. \& Giasson, J. (2006). La littératie familiale et les habiletés en conscience phonologique des enfants de maternelle. Éducation et francophonie, 34(2), 190-213. https://doi.org/10.7202/1079028ar
Résumé de l'article

Cet article présente les résultats d'une étude comparant les interventions parentales d'enfants de maternelle forts et faibles en conscience phonologique. Un échantillon de 88 élèves (44 dans chacun des groupes) fut constitué à partir des résultats de 234 élèves de maternelle qui furent évalués par une épreuve comprenant six tâches de conscience phonologique. Les mesures prises auprès des parents des enfants forts et faibles en conscience phonologique incluaient un questionnaire sur leurs interventions en conscience phonologique et des observations à la maison dans une activité de lecture d’un abécédaire. Les comparaisons des deux groupes révèlent que les parents d'enfants forts en conscience phonologique rapportent réaliser plus d'activités à la maison de conscience phonologique que les autres parents. De plus, lorsqu'ils sont observés pendant qu'ils lisent un abécédaire à leur enfant, ces parents font un plus grand nombre d'interventions et ils incitent plus souvent leur enfant à lire et à écrire (épeler) des mots.
Tous droits réservés (C) Association canadienne d'éducation de langue française, 2006
Cedocument est protégé par la loi sur le droit d'auteur. L'utilisation des services d'Érudit (y compris la reproduction) est assujettie à sa politique d'utilisation que vous pouvez consulter en ligne. 


\title{
La littératie familiale et les habiletés en conscience phonologique des enfants de maternelle
}

\author{
MONica BOUDREAU \\ Université du Québec à Rimouski, Québec, Canada \\ Lise SAINT-LAURENT \\ Université Laval, Québec, Canada \\ Jocelyne GIASSON \\ Université Laval, Québec, Canada
}

\section{RÉSUMÉ}

Cet article présente les résultats d'une étude comparant les interventions parentales d'enfants de maternelle forts et faibles en conscience phonologique. Un échantillon de 88 élèves ( 44 dans chacun des groupes) fut constitué à partir des résultats de 234 élèves de maternelle qui furent évalués par une épreuve comprenant six tâches de conscience phonologique. Les mesures prises auprès des parents des enfants forts et faibles en conscience phonologique incluaient un questionnaire sur leurs interventions en conscience phonologique et des observations à la maison dans une activité de lecture d'un abécédaire. Les comparaisons des deux groupes révèlent que les parents d'enfants forts en conscience phonologique rapportent réaliser plus d'activités à la maison de conscience phonologique que les autres parents. De plus, lorsqu'ils sont observés pendant qu'ils lisent un abécédaire à leur 
enfant, ces parents font un plus grand nombre d'interventions et ils incitent plus souvent leur enfant à lire et à écrire (épeler) des mots.

\section{ABSTRACT}

\section{Family Literacy and Phonological Awareness in Kindergarten Students}

Monica BOUDREAU

University of Quebec in Rimouski, Quebec, Canada

Lise SAINT-LAURENT

Laval University, Quebec, Canada

Jocelyne GIASSON

Laval University, Quebec, Canada

This article presents the results of a study comparing the interventions of parents of kindergarten students who had either strong or weak phonological awareness. A sample of 88 students (44 in each group) was formed using the results of an evaluation of 234 kindergarten students, which included six phonological awareness tasks. The parents of the students who had either strong or weak phonological awareness were given a questionnaire about the phonological awareness interventions and observations they made at home during a reading activity using an alphabet primer. The comparisons of the two groups revealed that the parents whose children had a strong awareness of phonetics do more phonological awareness activities at home with their children than the other parents do. Moreover, observations of these parents while they read an alphabet primer with their child, revealed that they made more interventions and encouraged their child to read and write (spell) words more often.

\section{RESUMEN}

\section{La literacia familiar y las habilidades en conciencia fonológica de los niños de preescolar}

Monica BOUDREAU

Universidad de Quebec en Rimouski, Quebec, Canadá

Lise SAINT-LAURENT

Universidad Laval, Quebec, Canadá

Jocelyne GIASSON

Universidad Laval, Quebec, Canadá

Este artículo presenta los resultados de un estudio que compara las intervenciones paternales de los niños con conciencia fonológica fuerte y débil. Se formó un muestreo de 88 alumnos (44 en cada uno de los grupos) a partir de los resultados de 
234 alumnos de preescolar evaluados con una prueba que comprendía seis ejercicios de conciencia fonológica. En lo que concierne a las familias de los niños con conciencia fonológica fuerte o débil, se incluyó un cuestionario sobre las intervenciones en conciencia fonológica así como observaciones en la casa durante una actividad de lectura de un abecedario. Las comparaciones entre los dos grupos muestran que los padres de niños con una conciencia fonológica fuerte realizan más actividades de conciencia fonológica en la casa que los otros padres. Además, cuando se les observa leyendo un abecedario a sus hijos, dichos padres intervienen más et los incitan con mayor frecuencia a leer, escribir (deletrear) las palabras.

\section{Introduction et problématique}

Les attitudes, les connaissances et les habiletés associées à la lecture et à l'écriture se développent chez l'enfant bien avant qu'il ne fréquente l'école (Ferreiro et Gomez Palacio, 1988). Cette sensibilisation au monde de l'écrit s'effectue sous l'in-

Par ailleurs, relativement peu d'études se sont penchées sur la littératie familiale et le développement de la conscience phonologique des enfants. Encore aujourd'hui, on sait peu de choses sur les activités parentales qui ont une incidence sur l'acquisition de cette habileté. fluence de l'environnement qui entoure l'enfant, dont le milieu socioculturel et la famille. On sait que les parents jouent un rôle de premier plan dans le développement des premières habiletés en lecture et en écriture de leur enfant. Ce rôle est depuis longtemps reconnu, mais aujourd'hui une nouvelle terminologie est d'usage : en effet, on parle maintenant de littératie familiale pour référer aux différentes interactions entre le parent et son enfant autour de la lecture et de l'écriture dans la vie de tous les jours (Britto et Brooks-Gunn, 2001). Un parent qui lit des histoires à son enfant, qui l'aide à écrire une carte d'anniversaire ou qui répond aux questions qu'il lui pose concernant l'écrit sont des exemples d'activités parentales caractéristiques de la littératie familiale. Les études dans ce domaine ont révélé que la littératie familiale influence les habiletés langagières de l'enfant (Bus, van IJzendoorn et Pellegrini, 1995; Sénéchal, LeFevre, Hudson et Lawson, 1996), ses connaissances sur les fonctions de l'écrit et sur les concepts reliés à l'écrit (Whitehurst et Lonigan, 1998), ses habiletés en écriture et sa connaissance du nom des lettres (Sénéchal et LeFevre, 2002).

Par ailleurs, relativement peu d'études se sont penchées sur la littératie familiale et le développement de la conscience phonologique des enfants. Encore aujourd'hui, on sait peu de choses sur les activités parentales qui ont une incidence sur l'acquisition de cette habileté. La présente étude s'intéresse aux interventions des parents en conscience phonologique avant l'entrée de leur enfant en première année. 


\section{La conscience phonologique et son développement}

Pour entrer dans le monde de l'écrit, l'enfant doit découvrir comment fonctionne le système alphabétique de la langue. Pour ce faire, il doit être sensibilisé au fait que les mots à l'oral sont composés d'unités plus petites. C'est ce qu'on appelle la conscience phonologique. La conscience phonologique peut se définir comme l'habileté à considérer les mots du langage oral indépendamment de leur signification et à manipuler les sons qui les constituent (Cunningham, 1990). La conscience phonologique constitue une composante du traitement phonologique, l'autre étant la mémoire phonologique (Lecocq, 1991; Sodoro, Allinder et Rankin-Erickson, 2002). La mémoire phonologique consiste dans l'habileté à coder les représentations du son dans la mémoire à court terme et à l'emmagasiner dans la mémoire à long terme (Sodoro et al., 2002). Les bons lecteurs se distingueraient des mauvais lecteurs sur cette habileté (Lecocq, 1991; Torgesen, Wagner, Rashotte, Rose, Lindamood, Conway et Garvan, 1999). Pour certains auteurs (Torgesen, 2000; Torgesen et al., 1999; Wagner et al., 1993, 1994, 1997), le traitement phonologique comprend une troisième composante : la rapidité de dénomination. Celle-ci consiste dans l'habileté à retrouver rapidement des informations dans la mémoire à long terme. Généralement, on l'évalue en demandant à l'enfant de nommer rapidement des couleurs, des images d'objets connus, des lettres ou des chiffres. Or, il y a une controverse à savoir si la rapidité de dénomination est une habileté phonologique ou non (Bower et Ishaik, 2003; Sodoro et al. 2002; Tannock, Martinussen et Frijters, 2000). En effet, plusieurs chercheurs (Badian, 1999; Bowers et Wolf, 1993; Manis, Doi et Bhadha, 2000; Wolf, Bowers et Biddle, 2000) la considèrent comme une habileté séparée du processus phonolo-

Dans les études des 20 dernières années, la conscience phonologique s'est révélée être un des meilleurs prédicteurs de la réussite en lecture. gique. Les tenants de cette dernière approche croient qu'un déficit en rapidité de dénomination signifie une lenteur dans le processus visuel possiblement d'origine neuropsychologique (Bowers et Ishaik, 2003; Sodoro et al., 2002; Wolf et al., 2000). Un déficit en rapidité de dénomination serait une explication possible des difficultés reliées à l'apprentissage de la lecture (Denckla et Rudel, 1976; Wolf et Bowers, 1999).

Dans les études des 20 dernières années, la conscience phonologique s'est révélée être un des meilleurs prédicteurs de la réussite en lecture (Snow, Burns et Griffin, 1998). Même si la conscience phonologique n'est pas suffisante à elle seule pour permettre l'apprentissage de la lecture, elle est indispensable à la mise en place des mécanismes de décodage. Être conscient que les mots sont composés de phonèmes facilite la compréhension du principe alphabétique et par le fait même influence le développement des habiletés d'identification de mots (Alegria et Morais, 1989; Gombert, 1992). Ainsi, des études comme celles de Stanovich, Cunningham et Cramer (1984), de Bradley et Bryant (1985), de Griffith et Olson, (1992), de Majsterek et Ellenwood (1995), de MacDonald et Cornwall (1995), de Troia (1999), de Demont (1994) et de Lecocq (1991) ont toutes mis en évidence un lien entre la conscience phonologique et la performance en lecture chez le lecteur débutant.

Plusieurs études ont tenté de cerner l'évolution de la conscience phonologique chez les enfants d'âge préscolaire. Entre trois ans et demi et quatre ans, l'enfant commence à être sensible aux rimes et il évolue ensuite dans sa maîtrise des unités 
linguistiques en passant de la rime à la syllabe et de la syllabe au phonème, et ce, autant en langue française qu'en langue anglaise (Alegria et Morais, 1979; Lecocq, 1991; Liberman, Shankweiler, Fisher et Carter, 1974; Maclean, Bryant et Bradley, 1987). De plus, la conscience phonologique semble se développer selon un continuum relatif aux différentes opérations linguistiques sollicitées (Adams, 1990). De façon générale, la tâche de catégorisation (Trouver parmi quatre mots celui qui ne commence pas par la même consonne initiale. Exemple : manteau, bureau, bœuf, ballon = manteau) est plus facile pour les enfants que celle de substitution (Isoler le son initial d'un mot et le remplacer par un son différent. Exemple : beau = chaud), qui est elle-même plus facile que celles de soustraction (Supprimer la consonne initiale d'un mot et prononcer le reste. Exemple : bœuf = œuf) et de segmentation (Taper le nombre de phonèmes qu'on entend dans un mot. Exemple : poisson = p/oi/ss/on) $($ Byrne et Fielding-Barnsley, 1990; Stanovich et al., 1984).

Étant donné son rôle primordial en lecture, la conscience phonologique a souvent fait l'objet de programmes d'entraînement. Les conclusions de deux métaanalyses portant sur l'entraînement à la conscience phonologique dans diverses langues, autres que le français, (Bus et van IJzendoorn, 1999; Ehri, Nunes, Willows, Schuster, Yaghoub et Shanahan, 2001) ont révélé que cet entraînement est plus efficace lorsqu'il est effectué dès la maternelle, lorsqu'il est combiné à l'enseignement des lettres de l'alphabet, lorsque les intervenants se consacrent à l'enseignement de une ou deux habiletés phonologiques (de préférence la fusion et la segmentation de phonèmes) et lorsque l'enseignement a lieu en petits groupes. L'entraînement à la conscience phonologique d'enfants francophones a aussi fait l'objet d'études. Des recherches réalisées en Belgique par Content, Morais, Alegria et Bertelson (1982), en France par Lecocq (1991) et au Québec par Couture (1998) et Brodeur (1994) ont confirmé les résultats des études anglophones à l'effet que les interventions en conscience phonologique effectuées auprès d'enfants francophones de maternelle pouvaient être efficaces.

\section{Les parents et la conscience phonologique}

Les conclusions des études concernant l'entraînement à la conscience phonologique des enfants dans un contexte expérimental nous amènent à poser la question du rôle éventuel des parents dans le développement de la conscience phonologique de leur enfant. Puisqu'il est possible d'entraîner les enfants en conscience phonologique, il est justifié de se demander jusqu'à quel point les interventions que les parents font spontanément à la maison ont un impact sur le développement des habiletés phonologiques de leur enfant. Cette question pourtant importante n'a pas reçu toute l'attention qu'elle mérite. Dans le domaine de la littératie familiale, l'attention a été largement concentrée sur l'activité, fort pertinente par ailleurs, qui consiste à lire des histoires aux enfants. Selon les résultats de l'ensemble des études, cette activité favorise surtout le développement du vocabulaire réceptif de l'enfant et le prépare à la lecture en facilitant son passage de l'oral à l'écrit (Bus et al., 1995). Cependant, la lecture d'histoires par les parents aurait peu d'influence sur le développement de la conscience phonologique de l'enfant (Aram et Levin, 2002; 
Evans, Shaw et Bell, 2000; Frijters, Baron et Brunello, 2000). Il est vrai cependant que Sénéchal et Lefevre (2002) ont identifié la présence d'un lien indirect entre la conscience phonologique des enfants et la lecture d'histoires par les parents.

Quelques rares études ont trouvé un lien entre le matériel disponible à la maison et le développement de la conscience phonologique des enfants. En se basant sur des entrevues menées auprès de parents de quatre enfants, Stuart (1990) suggère que l'utilisation de matériel tel les lettres mobiles peut faciliter le développement de la conscience phonologique. Plus récemment, Foy et Mann (2003) ont trouvé une corrélation significative entre la durée des activités de lecture reliées aux médias (jeux vidéo éducationnels, émissions de télévision et vidéos incluant le volet lecture) et les habiletés de conscience phonologique chez des enfants du préscolaire. Quelques autres études ont exploré le lien entre des activités concrètes des parents et la conscience phonologique. Ainsi, Maclean et ses collaborateurs (1987) ont trouvé des corrélations significatives entre la connaissance de comptines enfantines par les enfants de 3 ans 4 mois et le développement subséquent de leur conscience phonologique. Ces résultats suggèrent que les parents qui enseignent des comptines à leur enfant en bas âge favorisent le développement de sa conscience phonologique. La plupart des études qui ont identifié des interventions parentales pouvant être reliées à la conscience phonologique sont de nature exploratoire et ne comptent que

Étant donné l'importance des habiletés phonologiques dans le processus d'apprentissage de la lecture, il est nécessaire de mieux connaître ce que font les parents dans la vie de tous les jours au regard de la conscience phonologique. quelques sujets. Par exemple, Stuart (1990) a interviewé les parents de trois enfants forts en conscience phonologique et un parent d'enfant faible: les parents des enfants performants disent avoir sensibilisé très tôt leur enfant à des activités de langage autour de la rime et avoir encouragé leur enfant à écrire, alors que ces comportements ne sont pas présents dans la routine familiale de l'enfant plus faible en conscience phonologique. Smolkin et Yaden (1992) ont enregistré les parents de six enfants d'âge préscolaire ( 3 ans 6 mois à 4 ans 8 mois) pendant qu'ils faisaient la lecture d'un abécédaire à leur enfant : l'analyse des interactions parent-enfant autour de l'abécédaire suggère que cette activité peut aider les enfants à développer leur habileté à jouer avec les sons du langage. Ces résultats, qui reposent sur un nombre limité de sujets, doivent être confirmés par d'autres études. Il y a donc lieu de poursuivre les recherches afin d'identifier plus clairement les activités de littératie familiale en lien avec la conscience phonologique des enfants.

\section{Objectif de l'étude}

Étant donné l'importance des habiletés phonologiques dans le processus d'apprentissage de la lecture, il est nécessaire de mieux connaître ce que font les parents dans la vie de tous les jours au regard de la conscience phonologique. À la suite de la recension des écrits sur les activités de littératie familiale, une constatation majeure s'est imposée, soit l'absence presque complète ou du moins la rareté de recherches portant sur la stimulation à la conscience phonologique faite par les parents auprès de leur enfant. Pourtant, certains enfants entrent à la maternelle avec de grandes habiletés en conscience phonologique alors que chez d'autres cette habileté est pratiquement inexistante; on peut donc penser qu'il y a un lien entre ce qui se passe dans le milieu familial et le développement de la conscience phonologique des 
enfants. Cette étude a justement pour objectif de vérifier si les enfants de maternelle de différents niveaux de conscience phonologique viennent de familles qui se distinguent entre elles par les activités familiales reliées à la conscience phonologique. Plus spécifiquement, nous émettons l'hypothèse que les parents d'enfants forts en conscience phonologique font plus d'activités et d'interventions en conscience phonologique que ceux d'enfants faibles dans ce domaine. Par activités et interventions reliées à la conscience phonologique, nous entendons des activités et des interventions qui portent sur le langage oral et qui incitent l'enfant à faire une certaine manipulation des sons dans les mots.

\section{Méthode}

\section{Participantes et participants}

Les participantes et les participants sont des enfants francophones de maternelle cinq ans et un de leurs parents. Les enfants ont été recrutés dans 17 maternelles réparties dans sept écoles de la région de Québec situées dans des milieux socioéconomiques variés selon les indices de défavorisation fournis par le ministère de l'Éducation du Québec (2002). La sélection des sujets s'est effectuée en deux phases. La première phase a consisté à évaluer un grand nombre d'enfants de maternelle ( $N$ = 234: 111 filles et 123 garçons dont l'âge moyen est de 72,76 mois) afin d'obtenir deux groupes d'enfants ayant un écart maximal au plan de la conscience phonologique. Dans la deuxième phase, deux sous-groupes d'enfants ont été constitués à partir de l'échantillon initial, c'est-à-dire des enfants faibles $(n=49)$ en conscience phonologique et des enfants forts $(n=49)$ en conscience phonologique. Pour départir les groupes, le critère de sélection a été d'un demi écart-type de moins que la moyenne générale pour les élèves faibles en conscience phonologique et d'un demi écart-type de plus que la moyenne générale pour les élèves forts en conscience phonologique.

Afin de contrôler l'effet de l'enseignement en classe maternelle et du milieu socioéconomique, une procédure d'appariement des élèves a été utilisée. Les enfants faibles en conscience phonologique ont été pairés avec les forts en conscience phonologique en s'assurant que les élèves de la paire fassent partie du même groupe classe. Ce procédé fait en sorte que les enfants de la paire ont reçu à la maternelle la même stimulation à la conscience phonologique et qu'ils fréquentent une même école située dans un milieu socio-économique donné.

Des 49 paires ainsi constituées, cinq paires d'enfants n'ont pas été retenues pour les raisons suivantes: un parent s'est retiré de l'étude, un enfant refusait de collaborer aux épreuves et trois enfants ont obtenu des résultats très faibles à l'épreuve de rapidité de dénomination. Ainsi, l'échantillon final inclut 88 enfants : 44 enfants faibles en conscience phonologique (17 filles et 27 garçons; 72,23 mois) et 44 enfants forts en conscience phonologique (20 filles et 24 garçons; 72,95 mois). 


\section{Mesures}

\section{Auprès des enfants}

Une épreuve de conscience phonologique et une épreuve de rapidité de dénomination ont été utilisées.

Épreuve de conscience phonologique: L'épreuve de conscience phonologique utilisée porte sur différentes unités linguistiques et différentes opérations cognitives (Ziarko, De Koninck et Armand, 2003). Elle vise à évaluer la capacité des enfants à isoler et à manipuler les unités linguistiques suivantes: la syllabe, la rime et le phonème. Cette épreuve collective comporte six tâches composées de deux items d'entraînement et de quatre items expérimentaux: 1) identifier le phonème initial d'un mot cible (par exemple, l'enfant doit entourer l'image du mot qui commence comme lune: lampe, tasse, crayon), 2) catégoriser le phonème initial en identifiant le mot qui ne commence pas comme les deux autres (par exemple, l'enfant doit entourer l'image du mot qui ne commence pas comme les autres : sapin, citron, château), 3) catégoriser la rime en identifiant le mot qui ne se termine pas comme les deux autres (par exemple, l'enfant doit entourer l'image du mot qui ne finit pas comme les autres : couteau, serpent, éléphant), 4) supprimer la syllabe initiale identifiée d'un mot cible (par exemple, l'enfant doit entourer l'image du mot qui reste si on enlève le début de pompier: nez, pied, poire), 5) supprimer le phonème initial identifié d'un mot cible (par exemple, l'enfant doit entourer l'image du mot qui reste si on enlève le début de canne: dé, livre, âne) et 6) fusionner les phonèmes d'un mot prononcé de façon saccadée (par exemple, l'enfant doit entourer l'image qui montre ce que le robot a dit: [ch] [a]; chat, nid, main).

Chaque enfant reçoit un carnet dans lequel sont présentées les illustrations pour chacune des sous-épreuves. Il doit entourer l'illustration qui correspond à sa réponse pour chacun des items lus par l'examinateur. Un point est accordé pour chaque bonne réponse. Quatre points peuvent être obtenus à chacune des tâches permettant ainsi un résultat global de 24 points.

La validité de l'épreuve fut vérifiée avec les résultats obtenus par les 234 élèves du départ. Un coefficient alpha de .76 fut obtenu.

Épreuve de rapidité de dénomination (couleurs) : En se basant sur les travaux des chercheurs qui considèrent que la rapidité de dénomination est une habileté séparée du processus phonologique et qu'une lenteur dans ce domaine signifie qu'il y a un problème dans le processus visuel de nature neuropsychologique, nous avons voulu nous assurer que les élèves faibles en conscience phonologique n'avaient pas de déficits cognitifs d'une nature autre que phonologique. C'est ainsi que nous avons contrôlé l'effet de cette variable en évaluant tous les élèves et en éliminant de l'étude ceux étant très faibles en rapidité de dénomination.

Une épreuve de rapidité de dénomination des couleurs similaire à celle d'Evans et ses collaborateurs (2000) a été utilisée. Pour cette tâche, l'enfant doit nommer le plus rapidement possible cinq couleurs (rouge, jaune, bleu, noir et vert) qui lui sont présentées 50 fois de façon aléatoire sur un carton. Cette épreuve est administrée individuellement. Le score à cette épreuve est en corrélation significative avec la 
réussite en lecture (Sunseth et Bowers, 2002; Torgensen et al., 1999) et sa fidélité testretest après une période de 9 mois est de .78 (Evans et al., 2000).

\section{Auprès des parents}

Deux mesures relatives à la conscience phonologique ont été prises auprès des parents. Ces mesures ont été créées pour répondre aux besoins de la présente étude : il s'agit d'un questionnaire sur les activités parentales reliées à la conscience phonologique et d'une observation des interventions parentales dans une activité avec un abécédaire.

Questionnaire sur les activités reliées à la conscience phonologique: Le questionnaire élaboré vise à dresser le portrait le plus complet possible des activités parentales en lien avec la conscience phonologique. Dans le cadre de son élaboration, une première préoccupation a été d'identifier les activités susceptibles de favoriser le développement de la conscience phonologique des enfants du préscolaire. Des études comme celles de Brodeur, Valois, Dussault et Villeneuve (1999), de Maclean et ses collaborateurs (1987) et de Raz et Bryant (1990) ont servi à l'élaboration du questionnaire. Une deuxième préoccupation a été de formuler, de façon claire et précise, les questions portant sur les différentes composantes jugées pertinentes afin que les parents les comprennent facilement.

Le questionnaire comporte deux parties. La première partie est composée de 10 questions qui concernent l'habitude du parent d'attirer l'attention de son enfant sur les sons qui composent les mots pendant la lecture d'histoires ou pendant les activités quotidiennes. Ces questions sont présentées au tableau 2. Les réponses aux questions sont données sur une échelle de type Likert où les choix de réponses se distribuent de 1 à 5 . Le parent doit choisir parmi ces cinq choix: je ne pense jamais à faire cela, je pense rarement à faire cela, je pense quelquefois à faire cela, je pense souvent à faire cela et je pense très souvent à faire cela. La consistance interne du questionnaire a été établie au moyen du coefficient alpha de Cronbach. Le coefficient de consistance interne obtenu est de .72, ce qui est satisfaisant.

La deuxième partie du questionnaire comporte les quatre questions suivantes en lien avec la présence à la maison de matériel susceptible de favoriser les activités de conscience phonologique (Foy et Mann, 2003; Raz et Bryant, 1990; Stuart, 1990) : Avez-vous à la maison, les types de livres pour enfants suivants : abécédaire, recueil de comptines, de chansons, de poésie? À la maison, votre enfant joue-t-il avec des lettres mobiles? À la maison, votre enfant a-t-il à sa disposition du papier et des crayons? Avez-vous un ordinateur à la maison? Si oui, quels sont les logiciels avec lesquels votre enfant peut jouer avec des lettres? Ainsi, les parents devaient répondre par oui ou non concernant la présence à la maison de chacun de ces sept éléments de matériel pouvant favoriser la conscience phonologique. Le score maximal pour cette partie de questionnaire est donc de 7 points.

Observation des interventions parentales pendant la lecture d'un abécédaire : Une mesure directe des interventions en conscience phonologique a été prise auprès des parents. Ces derniers ont été invités à lire, devant un examinateur, une page d'abécédaire avec leur enfant. Certaines études nous permettent de penser que les 
activités autour de l'abécédaire sont de nature à attirer l'attention des enfants sur les sons présents dans les mots (Smolkin et Yaden, 1992).

Pour cette tâche, l'examinateur invite le parent à regarder le livre avec son enfant en procédant comme il le fait d'habitude. On insiste sur le fait qu'il n'y a pas de bonne ou de mauvaise réponse. On lui suggère de se servir de la page portant sur la première lettre du prénom de l'enfant. Par exemple, si l'enfant se prénomme Pascal, le parent est invité à lire la page de la lettre P. À la gauche de cette page, la lettre P est écrite en majuscule et en minuscule. En haut, toutes les lettres de l'alphabet sont représentées en majuscules. On retrouve toutes les lettres minuscules au bas de cette même page. Au centre, un enfant fait une action qui commence par la lettre P. Dans ce cas-ci, il peint et le mot peinture est écrit. En dessous de cette scène, trois mots commençant par la lettre P sont écrits et illustrés (pingouin, plume et poire).

L'examinateur consigne par écrit tout ce que le parent dit et fait. Quarante-deux comportements différents ont été observés chez les parents (voir annexe A) et ont été classés dans trois catégories : 1) les interventions reliées directement à la conscience phonologique (ex. : le parent demande à l'enfant de nommer un mot qui commence par la lettre cible), 2) les interventions reliées à l'identification des lettres (ex. : le parent demande à l'enfant de nommer les lettres d'un mot écrit sur la page) et 3) les interventions reliées à la lecture et à l'écriture de mots (ex. : le parent demande à l'enfant ce que fait un /b/ avec un /a/). La fiabilité de la classification des observations a été vérifiée par une procédure d'accord inter juges. Pour cette vérification, les juges sont partis des notes prises par les examinateurs et ont classé les interventions des parents dans l'une des trois catégories. Le pourcentage moyen d'accord inter juges calculé sur le total des comportements observés dans $20 \%$ des protocoles est de $97 \%$.

\section{Procédure}

La collecte de données s'est déroulée en fin d'année scolaire (avril, mai et juin). Les 234 enfants de l'échantillon initial ont été évalués collectivement par l'épreuve de conscience phonologique. Deux examinateurs ont participé à cette évaluation. Un menait l'activité alors que l'autre en supervisait le déroulement. Lors de la passation de l'épreuve, les illustrations furent présentées en format géant pour permettre aux enfants de bien suivre les consignes. Après la constitution des groupes d'enfants forts et faibles en conscience phonologique, les enfants retenus ont été évalués individuellement par l'épreuve de rapidité de dénomination. Trois sujets ont été retirés de l'étude parce qu'ils ont obtenu un résultat trop faible à cette épreuve. La moyenne des enfants à cette épreuve est de 58,92 secondes (écart-type=13,55) alors que ces trois sujets ont obtenu des scores extrêmes (entre 100 et 114 secondes). Les informations fournies par les enseignantes à leur sujet ont révélé qu'ils éprouvaient des difficultés d'apprentissage et de langage.

Les parents des enfants de l'échantillon ont été rencontrés à leur domicile. Lors de la prise de rendez-vous, il a été signalé aux parents que la rencontre serait effectuée avec la personne la plus impliquée dans le cheminement scolaire de l'enfant. Dans $88,6 \%$ des cas, le répondant était la mère, dans $10,2 \%$ le répondant était le père et dans $1,1 \%$ le répondant était une tante qui avait la responsabilité de l'enfant. Les 
rencontres se sont échelonnées sur une période de trois semaines.

Lors de la visite de l'examinateur à la maison, le parent était tout d'abord invité à réaliser l'activité avec l'abécédaire avec son enfant. Cette activité est d'une durée d'environ cinq minutes et est effectuée avant de soumettre le questionnaire aux parents afin de ne pas influencer leur comportement.

Par la suite, l'examinateur administrait le questionnaire sur les activités reliées à la conscience phonologique. Afin de minimiser l'effet possible du manque d'aisance en lecture de certains parents, les questions furent lues aux parents, mais le texte demeurait toutefois sous leurs yeux. De plus, afin de s'assurer que le parent comprenne bien les questions posées, un exemple concret du comportement évalué a été ajouté à chacune des questions.

\section{Résultats}

\section{Résultats à l'épreuve de conscience phonologique}

Le tableau 1 présente les résultats des deux groupes d'enfants obtenus aux six sous-épreuves phonologiques. Le score moyen en pourcentage pour le groupe d'enfants faibles en conscience phonologique est de $47,3 \%$ et de $89,8 \%$ pour les enfants forts en conscience phonologique. Les élèves faibles en conscience phonologique obtiennent les scores les plus bas à toutes les sous-épreuves phonologiques.

Tableau 1 : Moyennes, écarts-types et Funivariés obtenus à I'épreuve phonologique en fonction des groupes d'enfants faibles et forts en conscience phonologique

\begin{tabular}{|c|c|c|c|c|c|}
\hline \multirow[t]{2}{*}{$\begin{array}{l}\text { Épreuve de conscience } \\
\text { phonologique }\end{array}$} & \multicolumn{2}{|c|}{$\begin{array}{l}\text { Enfants faibles } \\
\qquad(n=44)\end{array}$} & \multicolumn{2}{|c|}{$\begin{array}{l}\text { Enfants forts } \\
\qquad(n=44)\end{array}$} & \multirow[b]{2}{*}{$\mathbf{F}$} \\
\hline & M & ÉT. & M & ÉT. & \\
\hline $\begin{array}{l}\text { Sous-épreuve } 1 \text { : } \\
\text { Identification du phonème initial }\end{array}$ & 43,8 & 24,3 & 82,5 & 17,5 & 73,6 \\
\hline $\begin{array}{l}\text { Sous-épreuve } 2 \text { : } \\
\text { Catégorisation du phonème initial }\end{array}$ & 30,0 & 22,0 & 83,5 & 23,5 & 121,5 \\
\hline $\begin{array}{l}\text { Sous-épreuve } 3 \text { : } \\
\text { Catégorisation de la rime }\end{array}$ & 38,0 & 28,3 & 91,5 & 14,3 & 125,4 \\
\hline $\begin{array}{l}\text { Sous-épreuve } 4 \text { : } \\
\text { Suppression de la syllabe initiale }\end{array}$ & 49,5 & 34,0 & 91,5 & 13,3 & 58,9 \\
\hline $\begin{array}{l}\text { Sous-épreuve } 5 \text { : } \\
\text { Suppression du phonème initial }\end{array}$ & 56,8 & 27,3 & 95,0 & 10,3 & $75,8^{*}$ \\
\hline $\begin{array}{l}\text { Sous-épreuve } 6 \text { : } \\
\text { Fusion de phonèmes }\end{array}$ & 66,5 & 24,8 & 95,0 & 10,3 & 49,8 \\
\hline Score total & 47,3 & 10,7 & 89,8 & 6,0 & $525,7^{2}$ \\
\hline
\end{tabular}




\section{Résultats au questionnaire sur les activités reliées à la conscience phonologique}

Le tableau 2 présente les résultats des deux groupes de parents à chacune des 10 questions de la première partie du questionnaire sur la stimulation à la conscience phonologique ainsi que leur score moyen total. Le score moyen total de stimulation en conscience phonologique est de 29,5 pour les parents des enfants forts en conscience phonologique et de 26,7 pour les parents des enfants faibles en conscience phonologique. Une analyse de variance univariée comparant le score total de stimulation en conscience phonologique des deux groupes de parents révèle une différence significative $[F(1,86)=4,41, p<.04 ;$ effet de grandeur $=.43]$ entre le résultat des parents des enfants faibles en conscience phonologique et ceux des enfants forts en conscience phonologique. Ainsi, les deux groupes de parents se distinguent en ce qui concerne la quantité totale de leurs activités de stimulation à la conscience phonologique. Une analyse corrélationnelle a été effectuée entre le score total de stimulation parentale et la conscience phonologique des enfants. Un coefficient $r$ de .31 fut obtenu ( $\mathrm{p}$ <.01), ce qui indique un lien modéré entre les deux variables. Les $\mathrm{F}$ univariés obtenus pour chacune des questions indiquent des différences significatives pour trois des 10 questions: la question $4[F(1,86)=4,22, p<0,05$; effet de grandeur $=0,43]$, la question $5[F(1,86)=10,47, p<0,01$; effet de grandeur $=0.65]$ et la question $7[F(1,86)=4,27, p<0,05$; effet de grandeur $=0,43]$. Les parents des enfants forts en conscience phonologique font plus de jeux avec les sons avec leur enfant (question 4), ils demandent plus souvent à leur enfant de trouver des mots qui riment avec les prénoms des membres de la famille (question 5) et de trouver des mots qui commencent par un son (question 7). Les analyses corrélationnelles révèlent que la conscience phonologique est en lien significatif mais faible avec les questions 4 ( $r=$ $.24, p \prec .05)$ et $7(r=.23, p \prec .05)$ et en lien modéré avec la question $5(r=.38, p \prec .01)$. 
Tableau 2: Moyennes et écarts-types obtenus au questionnaire sur les activités de stimulation à la conscience phonologique en fonction des deux groupes de parents

\begin{tabular}{|c|c|c|c|c|}
\hline \multirow[t]{2}{*}{ Questionnaire sur les activités de stimulation à la conscience phonologique } & \multicolumn{2}{|c|}{$\begin{array}{c}\text { Parents d'enfants } \\
\text { faibles } \\
(n=44)\end{array}$} & \multicolumn{2}{|c|}{$\begin{array}{c}\text { Parents d'enfants } \\
\text { forts } \\
(n=44)\end{array}$} \\
\hline & M & ÉT. & M & ÉT. \\
\hline $\begin{array}{l}\text { 1. Lorsque vous lisez un livre à votre enfant, attirez-vous son attention sur les mots } \\
\text { qui riment? }\end{array}$ & 2,7 & 1,3 & 2,9 & 1,1 \\
\hline $\begin{array}{l}\text { 2. Lorsque vous lisez un livre à votre enfant, attirez-vous son attention sur le son des } \\
\text { lettres? }\end{array}$ & 3,1 & 1,2 & 3,3 & 1,3 \\
\hline $\begin{array}{l}\text { 3. Lorsque vous lisez un livre à votre enfant, attirez-vous son attention sur les mots qui } \\
\text { commencent comme la première lettre de son prénom? }\end{array}$ & 3,5 & 1,3 & 3,3 & 1,3 \\
\hline 4. Dans la vie de tous les jours, vous arrive-t-il de jouer avec les sons avec votre enfant? & 2,2 & 1,1 & 2,7 & 1,4 \\
\hline $\begin{array}{l}\text { 5. Dans la vie de tous les jours, vous arrive-t-il de jouer à trouver des mots qui riment } \\
\text { avec les prénoms des membres de la famille? }\end{array}$ & 2,9 & 1,2 & 3,6 & 1,0 \\
\hline $\begin{array}{l}\text { 6. Dans la vie de tous les jours, vous arrive-t-il de demander à votre enfant de compter le } \\
\text { nombre de syllabes dans un mot en tapant dans les mains? }\end{array}$ & 1,9 & 1,2 & 1,8 & 1,0 \\
\hline $\begin{array}{l}\text { 7. Dans la vie de tous les jours, vous arrive-t-il de demander à votre enfant de trouver des } \\
\text { mots qui commencent par un son? }\end{array}$ & 2,7 & 1,1 & 3,3 & 1,3 \\
\hline $\begin{array}{l}\text { 8. Dans la vie de tous les jours, vous arrive-t-il de demander à votre enfant de faire un } \\
\text { mot en regroupant des syllabes? }\end{array}$ & 1,9 & 1,2 & 2,2 & 1,2 \\
\hline $\begin{array}{l}\text { 9. Dans la vie de tous les jours, vous arrive-t-il d'apprendre de nouvelles comptines à votre } \\
\text { enfant? }\end{array}$ & 2,4 & 1,3 & 2,8 & 1,2 \\
\hline $\begin{array}{l}\text { 10. Dans la vie de tous les jours, vous arrive-t-il de chanter ou de réciter avec votre enfant } \\
\text { des comptines qu'il a apprises ailleurs? }\end{array}$ & 3,4 & 1,0 & 3,6 & 1,0 \\
\hline Score moyen total & 26,7 & 6,4 & 29,5 & 6,2 \\
\hline
\end{tabular}

1 = je ne pense jamais à faire cela; 2 = je pense rarement à faire cela; 3 = je pense quelquefois à faire cela; 4 = je pense souvent à faire cela; 5 = je pense très souvent à faire cela.

\section{Résultats au questionnaire sur le matériel relié à la conscience phonologique}

Le tableau 3 présente les pourcentages calculés pour les deux groupes de parents concernant la présence dans l'environnement familial de matériel susceptible de favoriser les activités reliées à la conscience phonologique. Le score total moyen des parents des enfants forts en conscience phonologique est de 4,3 (écart-type $=1,5$ ) et celui des parents des enfants faibles en conscience phonologique est de 4,1 (écarttype $=1,5$ ), le score maximal étant de 7 points. L'analyse de variance menée sur ces scores est non significative, ce qui suggère qu'il n'y aurait pas plus de matériel relié à la conscience phonologique dans les foyers des enfants forts en conscience phonologique que dans ceux des enfants faibles en conscience phonologique. 
Tableau 3 : Pourcentages moyens obtenus aux questions sur la présence du matériel susceptible de favoriser les activités de conscience phonologique en fonction des deux groupes de parents

\begin{tabular}{|l|c|c|}
\hline $\begin{array}{l}\text { Questionnaire sur le matériel susceptible } \\
\text { de favoriser les activités de conscience } \\
\text { phonologique }\end{array}$ & $\begin{array}{c}\text { Parents d'enfants } \\
\text { faibles } \\
(\mathbf{n = 4 4 )}\end{array}$ & $\begin{array}{c}\text { Parents d'enfants } \\
\text { forts } \\
\text { (n= } \mathbf{4 4 )}\end{array}$ \\
\cline { 2 - 3 } $\begin{array}{l}\text { Types de livres } \\
\text { Abécédaire }\end{array}$ & 63,6 & $\%$ \\
\hline Recueil de comptines & 54,5 & 72,7 \\
Recueil de chansons & 50,0 & 50,0 \\
Recueil de poésie & 18,2 & 43,2 \\
\hline Autre matériel & & 25,0 \\
\hline Lettres mobiles & 59,1 & \\
Papiers et crayons & 95,5 & 63,6 \\
Ordinateur avec logiciels (lettres) & 70,5 & 100,0 \\
\hline
\end{tabular}

\section{Résultats à l'observation de l'activité avec l'abécédaire}

Le tableau 4 présente les résultats des parents concernant leurs interventions lors de l'activité avec l'abécédaire. Le score total pour l'ensemble des interventions est de 8,2 pour les parents des enfants forts en conscience phonologique et de 6,2 pour les parents des enfants faibles en conscience phonologique. En ce qui concerne les interventions directement en lien avec la conscience phonologique, la moyenne des parents des enfants forts en conscience phonologique est de 2,4 et celle des parents des enfants faibles en conscience phonologique, de 2,0. Les résultats reliés à l'identification des lettres de l'alphabet indiquent que les parents des enfants forts en conscience phonologique obtiennent une moyenne de 3,9 et ceux des enfants faibles en conscience phonologique une moyenne de 3,6. Les données associées à la lecture et à l'écriture de mots révèlent que les parents des enfants forts en conscience phonologique font deux fois plus d'interventions de ce type $(M=1,8)$ que les parents des enfants faibles en conscience phonologique $(M=0,7)$. 
Tableau 4 : Moyennes et écarts-types obtenus aux scores d'observation des interventions autour de l'abécédaire en fonction des deux groupes de parents

\begin{tabular}{|c|c|c|c|c|}
\hline \multirow[t]{2}{*}{ Activité d'animation avec l'abécédaire } & \multicolumn{2}{|c|}{$\begin{array}{c}\text { Parents d'enfants } \\
\text { faibles } \\
(n=44)\end{array}$} & \multicolumn{2}{|c|}{$\begin{array}{c}\text { Parents d'enfants } \\
\text { forts } \\
(n=44)\end{array}$} \\
\hline & M & ÉT. & M & ÉT. \\
\hline Comportements reliés à la conscience phonologique & 2,0 & 2,2 & 2,4 & 2,8 \\
\hline $\begin{array}{l}\text { Comportements reliés à l'identification des lettres } \\
\text { de l'alphabet }\end{array}$ & 3,6 & 2,5 & 3,9 & 2,8 \\
\hline $\begin{array}{l}\text { Comportements reliés à la lecture et à l'écriture } \\
\text { de mots }\end{array}$ & 0,7 & 2,2 & 1,8 & 2,0 \\
\hline Score moyen total des interventions & 6,2 & 3,6 & 8,2 & 4,4 \\
\hline
\end{tabular}

Nous avons tout d'abord comparé les parents des enfants forts et faibles en conscience phonologique en utilisant leur score moyen total d'interventions lors de l'activité. L'analyse de variance univariée révèle une différence significative $[F(1,86)=$ 5,57, $p<0,05$; effet de grandeur $=0,55$ ] entre les deux groupes de parents. Les parents des enfants forts en conscience phonologique font significativement plus d'interventions que les parents des enfants faibles en conscience phonologique lors de l'activité avec l'abécédaire. La corrélation entre les deux scores indique un lien significatif mais faible $(r=.26, p<.05)$

Des analyses de variance univariée ont été menées pour chacune des trois catégories de comportements observées. L'analyse portant sur les interventions directement associées à la conscience phonologique ne révèle pas de différence significative entre les parents des enfants faibles en conscience phonologique et ceux des enfants forts en conscience phonologique. Les mêmes résultats sont obtenus concernant les interventions reliées à l'identification des lettres de l'alphabet. Par contre, l'analyse de variance menée sur les scores des interventions reliées à la lecture et à l'écriture de mots révèle une différence significative $[F(1,86)=6,05, p<0,05$; effet de grandeur $=$ $0,52]$ entre les deux groupes. Les parents des enfants forts en conscience phonologique font significativement plus d'interventions reliées à la lecture et à l'écriture de mots que les parents des enfants faibles en conscience phonologique. Une analyse corrélationnelle menée sur les deux scores donne un coefficient $r$ de .37 ( $p<.01$ ) révélant un lien modéré entre les interventions parentales reliées à la lecture et à l'écriture de mots et la conscience phonologique des enfants. 


\section{Discussion}

Cette étude visait à comparer la stimulation parentale en conscience phonologique chez des enfants de maternelle forts et faibles en conscience phonologique. L'analyse des données révèle que les deux groupes d'enfants se distinguent par les

L'analyse des données révèle que les deux groupes d'enfants se distinguent par les activités reliées à la conscience phonologique qu'ils réalisent à la maison. Les parents des enfants habiles rapportent faire davantage d'interventions en conscience phonologique à la maison. activités reliées à la conscience phonologique qu'ils réalisent à la maison. Les parents des enfants habiles rapportent faire davantage d'interventions en conscience phonologique à la maison. Bien que dans un devis corrélationnel de type comparatif tel que celui utilisé dans cette étude, la relation entre les variables parentales et la conscience phonologique soit bidirectionnelle, on peut penser que plus l'enfant vit dans un environnement familial qui stimule sa conscience phonologique, plus ses habiletés dans ce domaine seront élevées. Cette interprétation de nos résultats va dans le sens des méta-analyses de Bus et van Ijzendoorn (1999) et de Ehri et ses collaborateurs (2001) qui concluent que des programmes d'intervention permettent le développement des habiletés phonologiques au préscolaire. Elle concorde également avec des études effectuées en milieu francophone comme celles de Brodeur (1994), de Content et ses collaborateurs (1982) et de Lecocq (1991) qui ont montré que les enfants qui reçoivent un entraînement à la conscience phonologique obtiennent des résultats significativement plus élevés à des épreuves phonologiques que ceux qui font partie des groupes témoins. L'autre interprétation à ces liens significatifs obtenus entre la stimulation parentale et la conscience phonologique est à l'effet que plus les enfants sont compétents en conscience phonologique, plus les parents sont stimulés ou encouragés à réaliser des activités de conscience phonologique. Pour éclairer le sens de ce lien entre la conscience phonologique des enfants et la stimulation parentale, des études avec un devis expérimental évaluant l'impact d'interventions parentales en conscience phonologiques devraient être menées dans le futur.

D'après les résultats du questionnaire sur la stimulation à la conscience phonologique, les parents des enfants performants en habiletés phonologiques se distinguent des autres parents, non seulement par la quantité des interventions, mais également par les types d'activités de conscience phonologique qu'ils disent privilégier à la maison. En effet, ces parents rapportent jouer plus souvent avec les sons avec leur enfant; ils se prêteraient également davantage à des activités qui portent sur la production de rimes et sur la production de mots qui commencent par un son cible. Ces résultats vont dans le même sens que ceux de Stuart (1990) qui a trouvé que les parents des enfants qui ont de bonnes habiletés phonologiques disent réaliser plus souvent que les autres parents des activités en lien avec la production de rimes et avoir sensibilisé très tôt leur enfant à des jeux de langage. Il est pertinent de signaler que les activités qui distinguent les deux groupes de parents portent sur la rime et le phonème en position initiale, alors qu'aucune différence significative n'est obtenue concernant les interventions impliquant la syllabe. En outre, les trois questions qui démarquent les deux groupes de parents concernent les activités effectuées dans la vie quotidienne. Aucune des questions portant sur les activités durant la lecture de livres n'a donné lieu à des différences significatives. Ce résultat corrobore 
ceux des études qui ont montré que la lecture d'histoires était plus propice au développement du vocabulaire qu'au développement de la conscience phonologique (Aram et Levin, 2002; Bus et al., 1995; Evans et al., 2000; Frijters et al., 2000; Sénéchal et Lefevre, 2002) .

Par ailleurs, d'après les résultats de la présente étude, la présence du matériel susceptible de favoriser les activités de conscience phonologique dans l'environnement familial serait équivalente dans les deux groupes de parents. Ces résultats suggèrent que ce n'est pas le matériel en lui-même qui est important pour stimuler la conscience phonologique mais plutôt la façon de l'utiliser. Il aurait peut-être été préférable, dans notre étude, de demander aux parents, non seulement s'ils possédaient le matériel, mais encore comment ils l'utilisaient et à quelle fréquence. Dans leur étude, Foy et Mann (2003) ont demandé aux parents combien de minutes par jour leur enfant jouait à des jeux éducatifs sur l'ordinateur et ont obtenu un lien significatif entre la lecture reliée aux médias (jeux vidéo éducationnels et émissions de télévision ou vidéos qui incluent un volet lecture) et les habiletés phonologiques des enfants.

Nos résultats ont également montré que les parents des enfants faibles en conscience phonologique et ceux des enfants forts en conscience phonologique se comportent différemment pendant la lecture d'un abécédaire. En effet, les deux groupes de parents se distinguent de façon significative par le nombre total d'interventions effectuées lors de l'activité. Les parents se distinguent également dans le type d'interventions qu'ils font pendant la lecture de l'abécédaire. Les résultats obtenus montrent que les parents des enfants forts en conscience phonologique font davantage d'interventions orientées vers la lecture ou l'écriture de mots que ceux des enfants faibles en conscience phonologique. Comme on sait que l'apprentissage de la lecture favorise le développement de la conscience phonologique (Alegria, Pignot et Morais, 1982; Perfetti, Beck, Bell et Hughes, 1987), une explication possible de nos résultats est à l'effet que les parents qui aident leurs enfants à lire et à écrire des mots leur permettent de faire des progrès dans l'acquisition de la lecture, ce qui a pour effet d'améliorer leurs compétences en habiletés phonologiques. Cette explication va dans le sens des résultats de Sénéchal et LeFèvre (2002) qui ont trouvé une relation indirecte entre les activités parentales et la conscience phonologique. Les activités des parents aideraient les connaissances alphabétiques qui, à leur tour, faciliteraient l'acquisition de la conscience phonologique. À nouveau, étant donné le devis corrélationnel utilisé dans cette étude, on ne peut conclure au lien causal entre le comportement des parents pendant la lecture de l'abécédaire et le niveau de conscience phonologique des enfants. L'explication alternative est toujours possible, les enfants plus forts en conscience phonologique incitent plus leurs parents que les enfants faibles à faire des interventions avec l'abécédaire et à lire et à écrire des mots avec eux.

Cependant, les résultats concernant les interventions orientées directement vers la conscience phonologique dans l'animation de l'abécédaire ne révèlent pas de différence entre les deux groupes de parents. Tout se passe comme si les parents des enfants forts en conscience phonologique intégraient les interventions en conscience phonologique dans la tâche plus complexe qu'est celle de la lecture ou de 
l'écriture de mots. Ce n'est pas tant qu'ils ne font pas beaucoup d'interventions en conscience phonologique, c'est plutôt qu'ils profitent de la situation pour intégrer leurs interventions d'une façon naturelle dans la lecture et l'écriture de mots.

Alors que cette étude révèle des liens significatifs entre les variables parentales et la conscience phonologique, il faut mentionner que la force de ces liens est de "faible " à " modéré », les effets de grandeur vont de .43 à .65 et les coefficients $\mathrm{r}$ vont de .24 à .38 . Ceci peut s'expliquer d'une part par le fait que d'autres facteurs influencent le développement de la conscience phonologique et d'autre part, par l'absence d'une grande différence entre les interventions des parents dans ce domaine. Si on veut maximiser l'effet des interventions parentales, des programmes à leur intention seraient possiblement nécessaires pour les sensibiliser à l'importance de la conscience phonologique dans la réussite scolaire en lecture et pour les encourager à stimuler davantage leur enfant.

confirment effectivement que les parents d'enfants forts en conscience phonologique font plus de jeux avec les rimes et les phonèmes que les autres parents, mais elles révèlent également qu'une bonne partie de la stimulation à la conscience phonologique se produit lorsque le parent aide son enfant à lire et à écrire des mots.

\section{Conclusion}

En conclusion, cette étude apporte des données nouvelles concernant le lien entre la conscience phonologique et les interventions parentales. Étant donné le rôle de cette habileté dans l'apprentissage de la lecture, ces résultats sont importants. La recherche doit se poursuivre par des études étudiant l'effet des interventions parentales en conscience phonologique. Cette recherche dans laquelle 139 parents ont été questionnés et observés ouvre la voie à ces études d'impact.

Par ailleurs, la présente étude a levé le voile sur les types d'interventions des parents en conscience phonologique. A priori, on peut être porté à penser qu'à la maison toutes les interventions en conscience phonologique se font sous forme de jeux. Nos données confirment effectivement que les parents d'enfants forts en conscience phonologique font plus de jeux avec les rimes et les phonèmes que les autres parents, mais elles révèlent également qu'une bonne partie de la stimulation à la conscience phonologique se produit lorsque le parent aide son enfant à lire et à écrire des mots. C'est grâce à l'observation des parents que nous sommes en mesure de tirer ces conclusions. Un des mérites de notre étude est donc d'avoir eu recours à l'observation directe des parents pour compléter les informations auto-rapportées des questionnaires. De plus, nous avons pris des précautions méthodologiques afin de contrôler l'effet de l'enseignement reçu en classe : nous avons pairé les enfants faibles en conscience phonologique avec les enfants forts en conscience phonologique de la même classe afin de nous assurer que les enfants de la paire avaient reçu le même enseignement en maternelle depuis le début de l'année scolaire et qu'ils provenaient du même milieu socio-économique. Nous avons aussi cherché à assurer une meilleure généralisation des résultats en choisissant des écoles appartenant à trois milieux socio-économiques différents (faible, moyen, élevé). 


\section{Références bibliographiques}

ADAMS, M. J. (1990). Learning to read: Thinking and learning about print. Cambridge, Mass. : MIT Press.

ALEGRIA, J. et MORAIS, J. (1979). Le développement de l'habileté d'analyse phonétique consciente de la parole et l'apprentissage de la lecture. Archives de Psychologie, 47, p. 251-270.

ALEGRIA, J. et MORAIS, J. (1989). Analyse segmentale et acquisition de la lecture, dans L. Rieben et C. Perfetti (Dir.), L'apprenti lecteur. Recherches empiriques et implications pédagogiques. Neuchâtel-Paris : Delachaux et Niestlé, p. 173-196.

ALLEGRIA, J., PIGNOT, E. et MORAIS, J. (1982). Phonetic analysis of speech and memory codes in beginning readers. Memory and Cognition, 10, p. 451-456.

ARAM, D. et LEVIN, I. (2002). Mother-child joint writing and storybook reading: Relations with literacy among low SES kindergartners. Merrill-Palmer Quarterly, 48, p. 202-224.

BADIAN, N.A. (1997). Dyslexia and the double deficit hypothesis. Annal of Dyslexia, 47 , p. 69-87.

BOWERS, P.G. et ISHAIK, G. (2003). RAN's contribution to understand reading disabilities, dans H.L. Swanson, K.R. Harris et S. Graham (Éds.), Handbook of learning disabilities. New York: The Guilford Press, p. 140-157.

BOWERS, P.G. et WOLF, M. (1993). Theoretical links between naming speed, precise timing mechanisms and orthographic skills in dyslexia. Reading Writing Interdisciplinary Journal, 5, p. 69-85.

BRADLEY, L. et BRYANT, P. (1985). Rhyme and reason in reading and spelling. Ann Arbor, MI : The University of Michigan Press.

BRITTO, P. R. et BROOKS-GUNN, J. (2001). The role of family literacy environments in promoting young children's emerging literacy skills. San Francisco : Jossey-Bass.

BRODEUR, M. (1994). L'effet de la connaissance du son de lettres sur l'apprentissage de la segmentation phonologique chez des enfants de maternelle. Thèse de doctorat inédite, Université Laval, Québec.

BRODEUR, M., VALOIS, P., DUSSAULT, M. et VILLENEUVE, P. (1999). Validation d'un questionnaire sur les croyances et les pratiques des enseignants de la maternelle à propos d'habiletés métaphonologiques. Revue canadienne de l'éducation, 24, p. 17-29.

BUS, A. G. et VAN IJZENDOORN, M. H. (1999). Phonological awareness and early reading: A meta-analysis of experimental training studies. Journal of Educational Psychology, 91, p. 403-414. 
BUS, A. G., VAN IJZENDOORN, M. H. et PELLEGRINI, A. D. (1995). Joint book reading makes for success in learning to read: A meta-analysis on intergenerational transmission of literacy. Review of Educational Research, 65, p. 1-21.

BYRNE, B. et FIELDING-BARNSLEY, R. (1990). Acquiring the alphabet principle: A case for teaching recognition of phoneme identity. Journal of Educational Psychology, 82, p. 805-812.

CONTENT, A., MORAIS, J., ALEGRIA, J. et BERTELSON, P. (1982). Accelerating the development of phonetic segmentation skills in kindergartners. Cahiers de psychologie cognitive, 2, p. 259-269.

COUTURE, C. (1998). Intervention en conscience phonologique auprès d'élèves de milieux défavorisés en classe maternelle. Thèse de doctorat inédite, Université Laval, Québec.

CUNNINGHAM, A. E. (1990). Explicit versus implicit instruction in phonemic awareness. Journal of Experimental Child Psychology, 50, p. 429-444.

DEMONT, É. (1994). Conscience phonologique, conscience syntaxique. Quel(s) rôle(s) dans l'apprentissage efficace de la lecture? dans J. Grégoire et B. Piérart (Dir.), Évaluer les troubles de la lecture. Les nouveaux modèles théoriques et leurs implications diagnostiques. Bruxelles : DeBoeck Université, p. 195-208.

DENCKLA, M.B. et RUDEL, R.G. (1976). Rapid automatized naming (R.A.N.): Dyslexia differentiated from other learning disabilities. Neuropsychologia, 14, p. 471-479.

EHRI, L. C., NUNES, S. R., WILLOWS, D. M., SCHUSTER, B. V., YAGHOUB-ZADEH, Z. et SHANAHAN, T. (2001). Phonemic awareness instruction helps children learn to read: Evidence from the National Reading Panel's meta-analysis. Reading Research Quarterly, 36, p. 250-287.

EVANS, M. A., SHAW, D. et BELL, M. (2000). Home literacy and their influence on early literacy skills. Canadian Journal of Experimental Psychology, 54, p. 65-75.

FERREIRO, E. et GOMEZ PALACIO, M. (1988). Lire-écrire comment s'y prennent-ils? Lyon: Centre régional de documentation pédagogique.

FOY, J. G. et MANN, V. (2003). Home literacy environment and phonological awareness in preschool children: Differential effects for rhyme and phonological awareness. Applied Psycholinguistics, 24, p. 59-88.

FRIJTERS, J. C., BARRON, R. W. et BRUNELLO, M. (2000). Direct and mediated influences of home literacy and literacy interest on prereaders' oral vocabulary and early written language skill. Journal of Educational Psychology, 92, p. 466-477.

GOMBERT, J. E. (1992). Activité de lecture et activités associées, dans M. Fayol, J. E. Gombert, P. Lecocq, L. Sprenger-Charolles et D. Zagar (Eds.), Psychologie cognitive de la lecture. Paris : Presses Universitaires de France, p. 107-140. 
GRIFFITH, P. L. et OLSON, M. W. (1992). Phonemic awareness helps beginning readers break the code. The Reading Teacher, 45, p. 516-523.

LECOCQ, P. (1991). Apprentissage de la lecture et dyslexie. Liège : Mardaga.

LIBERMAN, I. Y., SCHANKWEILER, D., FISCHER, F. W. et CARTER, B. (1974). Explicit syllable and phoneme segmentation in the young child. Journal of Experimental Child Psychology, 18, p. 201-212.

MACDONALD, G. W. et CORNWALL, A. (1995). The relationship between phonological awareness and reading and spelling achievement eleven years later. Journal of Learning Disabilities, 28, p. 523-527.

MACLEAN, M., BRYANT, P. et BRADLEY, L. (1987). Rhymes, nursery rhymes, and reading in early childhood. Merrill-Palmer Quarterly, 33, p. 255-281.

MAJSTEREK, D. J. et ELLENWOOD, A. E. (1995). Phonological awareness and beginning reading: evaluation of a school-based screening procedure. Journal of Learning Disabilities, 28, p. 449-456.

MANIS, F.R., DOI, L.M. et BHADHA, G. (2000). Naming speed, phonological awareness, and orthographic knowledge in second graders. Journal of Learning Disabilities, 33, p. 325-333.

MINISTÈRE DE L'ÉDUCATION DU QUÉBEC. (2002). Indice de milieu socio-économique par école. Données 2002-2003. [www.meq.gouv.qc.ca]

PERFETTI, C. A., BECK, I., BELL, L. C. et HUGHES, C. (1987). Phonemic knowledge and learning to read are reciprocal: A longitudinal study of first grade children. Merrill-Palmer Quarterly, 33, p. 283-319.

RAZ, I. S. et BRYANT, P. (1990). Social background, phonological awareness and children's reading. British Journal of Developmental Psychology, 8, p. 209-225.

SENECHAL, M. et LEFEVRE, J. (2002). Parental involvement in the development of children's reading skill: A five-year longitudinal study. Child Development, 73, p. 445-460.

SÉNÉCHAL, M., LEFEVRE, J., HUDSON, E. et LAWSON, E. P. (1996). Knowledge of storybooks as a predictor of young children's vocabulary. Journal of Educational Psychology, 88, p. 520-536.

SMOLKIN, L. B. et YADEN, D. B. (1992). O is for Mouse: First encounters with the alphabet book. Language Arts, 69, p. 432-441.

SNOW, C., BURNS, M. et GRIFFIN, P. (1998). Preventing Reading Difficulties in Young Children. Washington, D.C. : National Academy Press.

SODORO, J., ALLINDER, R. M. et RANKIN-ERICKSON, J. L. (2002). Assessment of phonological awareness: Review of methods and tools. Educational Psychology Review, 14, p. 223-260. 
STANOVICH, K. E., CUNNINGHAM, A. E. et CRAMER, B. B. (1984). Assessing phonological awareness in kindergarten children: Issues of task comparability. Experimental Child Psychology, 38, p. 175-190.

STUART, M. (1990). Factors influencing word recognition in pre-reading children. British Journal of Psychology, 81, p. 135-146.

SUNSETH, K. et BOWERS, P.G. (2002). Rapid naming and phonemic awareness: Contributions to reading, spelling, and orthographic knowledge. Scientific Studies of Reading, 6, p. 401-429.

TANNOCK, R., MARTINUSSEN, R. et FRIJTERS, J. (2000). Naming speed performance and stimulant effects indicate effortful, semantic processing deficits in attentiondeficit/hyperactivity disorder. Journal of Abnormal Child Psychology, 28, p. 237-252.

TORGESEN, J.K. (2000). Individual differences in response to early intervention in reading: The lingering problem of treatment resister. Learning Disabilities Research \& Practice, 15(1), p. 55-64.

TORGESEN, J. K., WAGNER, R. K., RASHOTTE, C. A., ROSE, E., LINDAMOOD, P., CONWAY, T. et GARVAN, C. (1999). Preventing reading failure in young children with phonological processing disabilities: Group and individual responses to instruction. Journal of Educational Psychology, 91, p. 579-593.

TROIA, G. A. (1999). Phonological awareness intervention research: A critical review of the experimental methodology. Reading Research Quarterly, 34, p. 28-52.

WAGNER, R.K., TORGESEN, J.K., LAUGHON, P.l., SIMMONS, K. et RASHOTTE, C.A. (1993). The development of young readers' phonological processing abilities. Journal of Educational Psychology, 85, p. 83-103.

WAGNER, R.K., TORGESEN, J.K. et RASHOTTE, C.A. (1994). The development of reading-related phonological processing abilities: New evidence of bidirectional causality from a latent variable longitudinal study. Developmental Psychology, 30, p. 73-87.

WAGNER, A.K., TORGESEN, J.K., RASHOTTE, C.A., HECHT, S.A., BARKER, T.A., BURGESS, S.R. DONOHUE, J. et GARON, T. (1997). Changing relations between phonological processing abilities and word-level reading as children develop from beginning to skilled readers: A five-year longitudinal study. Developmental Psychology, 33, p. 468-479.

WHITEHURST, G. J. et Lonigan, C. J. (1998). Child development and emergent literacy. Child Development, 69, p. 848-872.

WOLF, M. et BOWERS, P.G. (1999). The double-deficit hypothesis for the developmental dyslexias. Journal of Educational Psychology, 91, p. 425-438.

WOLF, M., BOWERS, P.G. et BIDDLE, K. (2000). Naming-speed processes, timing, and reading: A conceptual review. Journal of Learning Disabilities, 33, p. 387-407. 
ZIARKO, H., DE KONINCK, Z. et ARMAND F. (2003). Profils cognitivo-langagiers d'élèves québécois francophones à la fin de la maternelle, dans $\mathrm{M}$. N . Romdhane, J. É. Gombert et M. Belajouza (Eds.), L'apprentissage de la lecture. Perspectives comparatives. Presses Universitaires de Rennes, Centre de Publications Universitaires de Tunis, p. 297-319.

\section{Annexe}

\section{Grille d'observation pour l'activité avec l'abécédaire}

\begin{tabular}{|c|c|}
\hline Comportements observés & \multirow{2}{*}{ Fréquence } \\
\hline 1- Comportements reliés aux lettres & \\
\hline a) Identifier des lettres isolées dans la page & \\
\hline 1. Demande à l'enfant s'il connaît le nom de la lettre cible. & \\
\hline 3. Nomme le nom de la lettre cible. & \\
\hline 2. Demande à l'enfant s'il connaît le nom d'une autre lettre. & \\
\hline 4. Nomme le nom d'une autre lettre. & \\
\hline b) Identifier des lettres dans un mot & \\
\hline 5. Demande de nommer la première lettre d’un mot qui est écrit sur la page. & \\
\hline 6. Demande de montrer où est la lettre cible dans un mot de la page. & \\
\hline 7. Demande de nommer les lettres d'un mot qui est écrit sur la page. & \\
\hline 8. Nomme les lettres d'un mot qui est écrit sur la page. & \\
\hline $\begin{array}{l}\text { 9. Aide l'enfant à identifier une lettre (lettre dans un mot, lettre dans la chaîne } \\
\text { alphabétique, etc.). }\end{array}$ & \\
\hline c) Autres interventions ou activités autour des lettres & \\
\hline $\begin{array}{l}\text { 10. - Chaîne alphabétique (demande de nommer les lettres de l'alphabet en ordre) } \\
\text { - Lettres minuscules et majuscules (pointe une lettre et demande si elle est minus- } \\
\text { cule ou majuscule). } \\
\text { - Jeux autour des lettres (demande de nommer toutes les lettres rouges, demande } \\
\text { de montrer les lettres de son prénom, cache des lettres et l'enfant doit les identi- } \\
\text { fier, fait semblant de se tromper en disant une lettre pour que l'enfant la nomme } \\
\text { correctement, demande de lui montrer où est le m, demande de compter tous } \\
\text { les r sur la page, etc.). }\end{array}$ & \\
\hline 2. Comportements reliés à la conscience phonologique & \\
\hline 11. Demande à l'enfant s'il connaît le son de la lettre cible. & \\
\hline 12. Fait le son de la lettre cible. & \\
\hline 13. Demande à l'enfant s'il connaît le son d'une autre lettre. & \\
\hline 14. Fait le son d'une autre lettre. & \\
\hline $\begin{array}{l}\text { 15. Attire l'attention de l'enfant sur le fait que les mots de la page commencent tous } \\
\text { par la même lettre ou le même son. }\end{array}$ & \\
\hline
\end{tabular}




\begin{tabular}{|c|c|}
\hline 16. Invite l'enfant à faire le lien entre la lettre cible et son prénom. & \\
\hline 17. Nomme un mot qui commence par la lettre ou le son cible. & \\
\hline $\begin{array}{l}\text { 18. Nomme un mot et demande à l'enfant s'il commence par la lettre cible ou le } \\
\text { son cible. }\end{array}$ & \\
\hline 19. Nomme un mot et demande à l'enfant s'il contient la lettre cible ou le son cible. & \\
\hline 20. Invite l'enfant à nommer des sons qu'il entend dans un mot. & \\
\hline 21. Invite l'enfant à trouver un mot qui rime avec un autre mot. & \\
\hline 22. Nomme un mot qui rime avec un autre mot. & \\
\hline $\begin{array}{l}\text { 23. Invite l'enfant à trouver un mot qui commence par une autre lettre ou un autre son } \\
\text { (que son prénom). }\end{array}$ & \\
\hline $\begin{array}{l}\text { 24. Nomme un mot qui commence par une autre lettre ou un autre son (que le } \\
\text { prénom de l'enfant). }\end{array}$ & \\
\hline 25. Dit que le mot commence par tel son. & \\
\hline 26. Dit que le mot contient tel son. & \\
\hline $\begin{array}{l}\text { 27. Invite l'enfant à donner le nom d'une lettre en pensant à un mot qu'il connaît et } \\
\text { qui commence par cette lettre. }\end{array}$ & \\
\hline $\begin{array}{l}\text { 28. Aide l'enfant à identifier l'image en attirant son attention sur le premier son } \\
\text { du mot. }\end{array}$ & \\
\hline $\begin{array}{l}\text { 29. Lit le mot (ou dit le mot) en insistant sur le début du mot (premier son ou } \\
\text { première syllabe). }\end{array}$ & \\
\hline 3- Comportements reliés à la lecture et à l'écriture d'un mot & \\
\hline 30. Demande à l'enfant de lire un mot. & \\
\hline 31. Aide l'enfant à lire un mot. & \\
\hline 32. Lit le mot en le séparant en syllabes. & \\
\hline 33. Lit le mot en identifiant les sons. & \\
\hline 34. Demande ce que fait un $b$ avec un a, etc. & \\
\hline 35. Dit qu'un b avec un a fait ba. & \\
\hline 36. Demande quel son fait on, ai, etc. & \\
\hline 37. Dit que on fait /on/, ph fait / $f /$, etc. & \\
\hline 38. Demande de copier sur une feuille un mot de la page. & \\
\hline $\begin{array}{l}\text { 39. Demande de nommer la première lettre ou le premier son d'un mot qui n'est pas } \\
\text { écrit sur la page. }\end{array}$ & \\
\hline $\begin{array}{l}\text { 40. Demande de nommer les lettres ou les sons d'un mot qui n'est pas écrit sur } \\
\text { la page. }\end{array}$ & \\
\hline 41. Nomme les lettres d'un mot qui n'est pas écrit sur la page. & \\
\hline 42. Dit un mot en syllabes et demande à l'enfant de l'écrire sur une feuille. & \\
\hline
\end{tabular}

